\title{
Frailty Syndrome in Biliary Tract Cancer Patients: Prevalence and Associated Factors
}

\author{
Panita Limpawattana ${ }^{1}$, Kosin Wirasorn ${ }^{2}$, Aumkhae Sookprasert ${ }^{2}$, Kittisak \\ Sawanyawisuth $^{3}$, Attapol Titapun ${ }^{4}$, Vor Luvira ${ }^{4}$, Narong Khuntikeo ${ }^{4}$, Jarin \\ Chindaprasirt ${ }^{2 *}$
}

\begin{abstract}
Background: Frailty, a risk of unfavorable outcomes from disability to adapt to stressors, is common in the elderly but could occur in younger patients with cancer. This study focused on the prevalence of frailty in biliary tract cancer (BTC) and associated factors. Methods: A total of 75 BTC patients receiving chemotherapy from January 2016 to September 2017 were enrolled. Frailty was diagnosed by the FRAIL (Fatigue, Resistance, Ambulation, Illnesses, and Loss of weight) scale. Univariate and multivariate regression analyses were used to analyze factors associated with frailty. Results: The prevalence rates of frailty and pre-frailty were 12\% (9 cases) and $60 \%$ (45 cases). According to multivariate logistic analysis, three factors were associated with frailty, including old age, high body mass index (BMI), and history of liver surgery. The adjusted odds ratios of the factors were $1.12(95 \% \mathrm{CI}: 1.01,1.24), 1.28(95 \% \mathrm{CI}: 1.01$, 1.64), and 6.16 (95\%CI: 1.11, 34.16), respectively. Conclusion: Frailty is not uncommon in BTC patients. Old age, high BMI, and history of liver surgery are important risk factors for frailty.
\end{abstract}

Keywords: FRAIL scale- Cholangiocarcinoma- prevalence- Pre-frail

Asian Pac J Cancer Prev, 20 (5), 1497-1501

\section{Introduction}

Frailty is characterized by a loss of ability to adapt to stress as a result of diminished functional reserves (Angulo et al., 2016). The decline is a result of multisystem involvement and increases the risk of poor outcomes (Fried et al., 2001). The prevalence of frailty is about $10 \%$ in the general population who aged 65 and over and $25-40 \%$ in those aged more than 85 (Collard et al., 2012; Lee et al., 2016; Kojima et al., 2017). It has become increasingly established as an important health issue in older adults and in cancer.

Frailty is common but not limited to advanced age. Younger patients, especially those with cancer, can have frailty as well. Both the disease and its treatments could be the additional stressors that increase the risk of adverse events (Ethun et al., 2017). It is one of the important issues in cancer patients who are undergoing surgery, radiation, and chemotherapy (Kristjansson et al., 2010; Handforth et al., 2015; Ethun et al., 2017). Frail patients are more likely to develop postoperative complications, adverse events from chemotherapy, shorter progression-free and overall survival (Handforth et al., 2015; Gani et al., 2017).

Biliary tract cancer is an aggressive liver tumor with poor survival outcome. Patients usually suffer from obstructive jaundice, malnutrition, and abdominal pain. The benefit of chemotherapy is modest (Sookprasert et al., 2012). Better knowledge of frailty in this setting will guide the treatment decisions to balance the risk and benefit of chemotherapy in biliary tract cancer. This study aimed to study the prevalence and factors associated with frailty in patients with biliary tract cancer who received chemotherapy.

\section{Materials and Methods}

This prospective study included biliary tract cancer patients who were planned to receive chemotherapy at the chemotherapy unit, Khon Kaen University, Thailand from January 2016 to September 2017. All patients were Eastern Cooperative Oncology Group (ECOG) performance status of 0-1. Patients were excluded if they had other active medical illnesses and patients who were unable to perform the Dual-energy X-ray absorptiometry (DXA) or conditions that would affect the DXA results.

\section{Definition}

Frailty syndrome

Frailty syndrome was defined by the FRAIL scale, which is a validated screening tool (Abellan van Kan et 
al., 2008). It consists of a 5-item scale:

F: Fatigue (feeling exhausted most of the time)

R: Resistance (limitations climbing 1 flight of stairs)

A: Ambulation (inability to walk alone for $100 \mathrm{~m}$ )

I: Illness (five to 11 of these diseases: hypertension, diabetes mellitus, cancer, chronic lung disease, heart attack, congesting heart failure, angina, asthma, arthritis, stroke, kidney disease)

L: Loss of weight (unintentional weight loss of $>5 \%$ over a past year)

One score was for each question. Patients who scored one or two were defined as pre-frail, while those who had three or more of the five criteria were defined as frail.

\section{Instruments}

Dual-energy x-ray absorptiometry (DXA) (General Electric [Lunar-Prodigy] model) was used to measure muscle, fat, and bone mass.

\section{Sample size calculation}

Sample size calculation was based on the primary objective of the study. Since there was no study regarding the prevalence of frailty in cholangiocarcinoma patients receiving chemotherapy, the estimated prevalence of $13 \%$ was derived from a previous study in colon cancer (Kristjansson et al., 2012). With the confidence of 95\%, the estimated sample size was 68 .

\section{Statistical analysis}

Baseline and clinical characteristics were analyzed using descriptive statistics. To compare the differences in numbers and proportions between frail and fit groups, Wilcoxon rank sum and Fisher's exact tests were applied.

Factors associated with frailty were evaluated with univariate logistic regression analysis. All clinically significant variables were included in subsequent multivariate logistic regression analyses. Factors in the final model were predictors for frailty if the p-value was less than 0.05 . Analytical results were presented as adjusted odds ratio (OR) and $95 \%$ confidence intervals.

Survival analysis was performed using the Kaplan-Meier method and log-rank test. All data analysis was performed using STATA software (StataCorp LP, College Station, TX, USA). This study was approved by the Khon Kaen University Faculty of Medicine Ethics Committee as instituted by the Declaration of Helsinki (Number HE581333).

\section{Results}

A total number of 75 biliary tract cancer patients were included. The prevalence of frailty and pre-fail state according to FRAIL scale was $12 \%$ (9 cases) and 60\% (45 cases). Prevalence of frailty syndrome increased with age; $7.8 \%, 15 \%$, and $50 \%$ in patients aged $<65,65-74, \geq 75$ years respectively (Figure 1).

Frail patients had a mean age of 64.22 years and a mean body mass index (BMI) of $23.20 \mathrm{~kg} / \mathrm{m}^{2} ; 6$ of the patients were male. The not frail subjects had a mean age of 58.56 years and a mean BMI of $21.43 \mathrm{~kg} / \mathrm{m}^{2} ; 52$ subjects were male (Table 1). The frail group had a history
Table 1. Comparison of Clinical Factors between Patients without and with Frailty Syndrome

\begin{tabular}{lccc}
\hline Factors & $\begin{array}{c}\text { Frail } \\
(\mathrm{n}=9)\end{array}$ & $\begin{array}{c}\text { Not frail } \\
(\mathrm{n}=66)\end{array}$ & p-value \\
\hline Age, years & $64.22(8.42)$ & $58.56(8.56)$ & 0.066 \\
Male, $\mathrm{n}(\%)$ & $6(66.67)$ & $52(78.79)$ & 0.415 \\
Weight, kg & $59.89(8.68)$ & $56.38(8.90)$ & 0.270 \\
Height, $\mathrm{m}$ & $161.11(6.01)$ & $162.30(6.28)$ & 0.593 \\
BMI, kg/m ${ }^{2}$ & $23.20(4.07)$ & $21.43(3.37)$ & 0.155 \\
Muscle mass, (kg) & $38.84(6.33)$ & $46.13(43.60)$ & 0.620 \\
Fat mass, (kg) & $16.43(7.34)$ & $12.61(6.85)$ & 0.124 \\
White blood cell & $8155(3542)$ & $8631(3689)$ & 0.717 \\
count, cells/mm ${ }^{3}$ & & & \\
Stage IV, n (\%) & $8(88.89)$ & $56(84.85)$ & 0.663 \\
Liver surgery, $\mathrm{n}(\%)$ & $6(66.67)$ & $20(30.30)$ & $0.032^{*}$ \\
\hline BMI, body mass index & & &
\end{tabular}

of liver surgery significantly more than the control group (66.67\% vs $30.30 \%)$.

After adjustment by multivariate logistic regression, the final model was comprised of four factors (Table 2). Three factors were associated with frailty syndrome, including old age, high BMI, and history of liver surgery. The adjusted ORs of the factors were 1.12 (95\%CI: 1.01 , 1.24), 1.28 (95\%CI: 1.01, 1.64), and 6.16 (95\% CI: 1.11, $34.16)$, respectively.

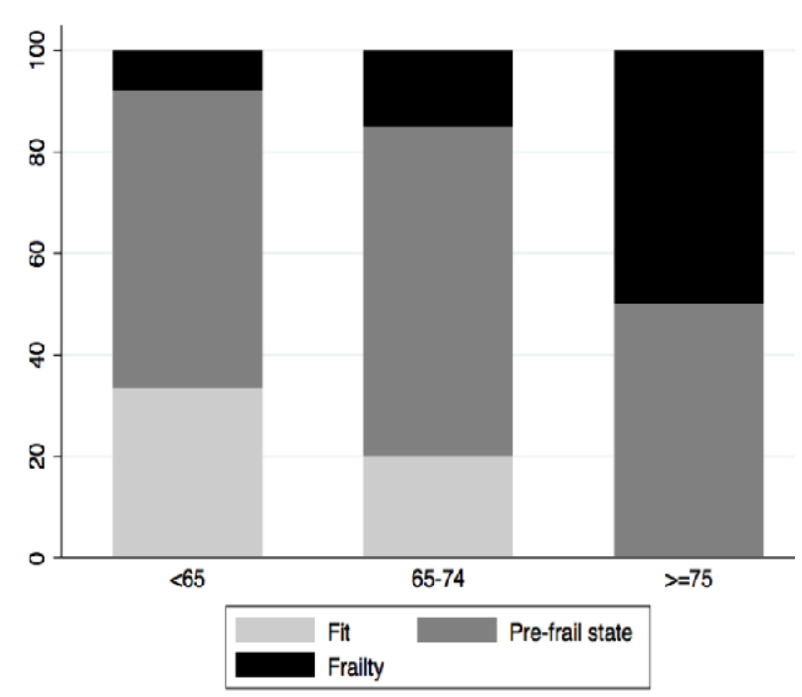

Figure 1. Prevalence of Frailty Syndrome According to Age-Group

Table 2. Univariate and Multivariate Logistic Regression Analyses of Factors Associated with Frailty Syndrome

\begin{tabular}{lcc}
\hline Factors & Crude OR $(95 \% \mathrm{CI})$ & Adjusted OR (95\% CI) \\
\hline Age & $1.080(0.992,1.176)$ & $1.120(1.011,1.240)$ \\
BMI & $1.149(0.947,1.395)$ & $1.285(1.005,1.643)$ \\
Female & $1.857(0.412,8.376)$ & $1.951(0.328,11.594)$ \\
$\begin{array}{l}\text { Liver } \\
\text { surgery }\end{array}$ & $4.600(1.045,20.247)$ & $6.160(1.111,34.160)$ \\
\hline
\end{tabular}

OR, odds ratio; CI, confidence interval; BMI, body mass index 

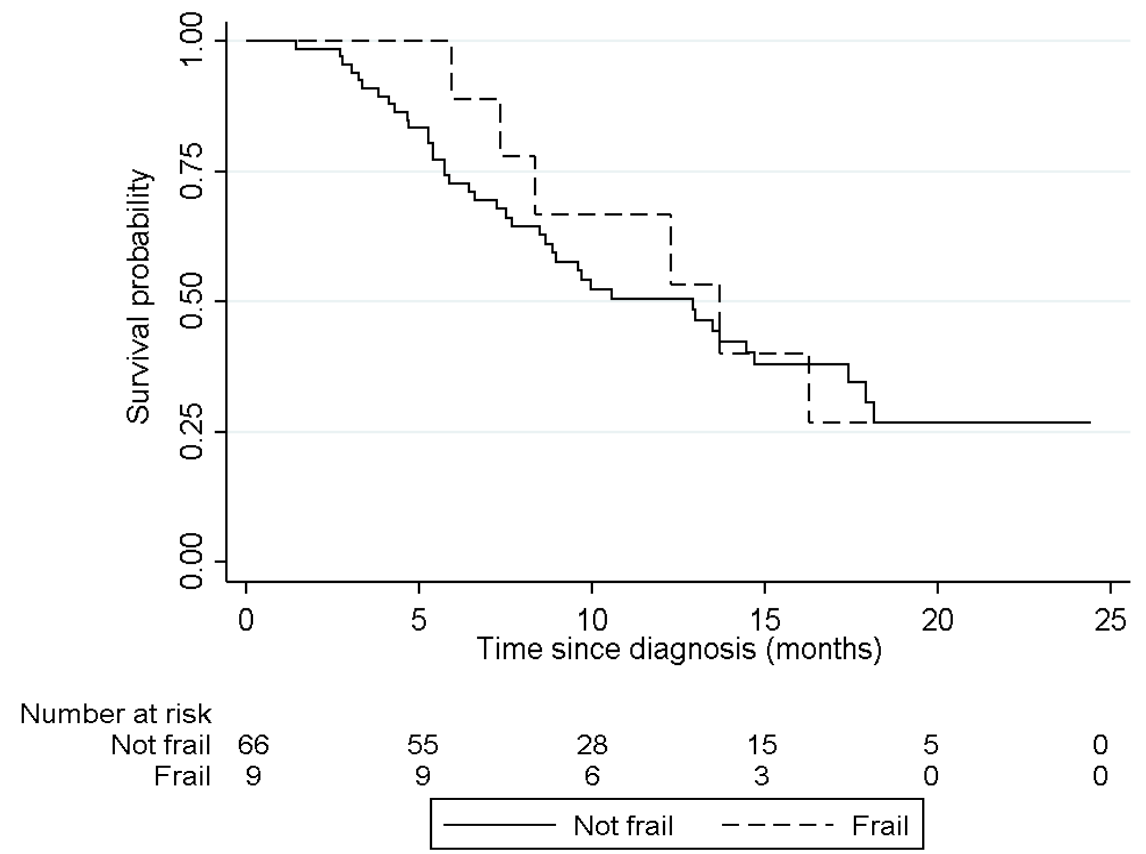

Figure 2. Survival Curve Comparing Fit and Frail Patients

As shown in Figure 2, frailty syndrome is not associated with poor survival outcome. The median survival time in frail patients were comparable to fit patients; 13.7 vs 12.9 months (HR 0.86; 95\%CI 0.36-2.02). Nevertheless, for fit patients, those who received carboplatin-based regimen had significantly shorter survival compared to those who received cisplatin-based chemotherapy (Figure 3); 8.5 vs 14.7 months (HR 2.30; 95\% CI 1.19-4.47, p-value 0.013).

\section{Discussion}

Frailty syndrome is common in biliary tract cancer patients. The prevalence of frailty in the cohort was $12 \%$. Older age, higher BMI, and history of liver surgery were independent factors associated with frailty.
The prevalence of frailty in this study was comparable to other studies using the same phenotype model reported in gynecological patients and those older patients who underwent surgery (Courtney-Brooks et al., 2012; Kristjansson et al., 2012). It is less common compared to the systematic review which included patients with all primary sites (Handforth et al., 2015). The possible explanation is the differences in study population because this study included patients with all ages and only patients who were suitable for chemotherapy; implying good ECOG performance status, while other studies included older adults with various functional statuses.

Older age suggests a high risk of frailty similar to several studies both in cancer and non-cancer patients. Patients who underwent liver surgery had a high risk

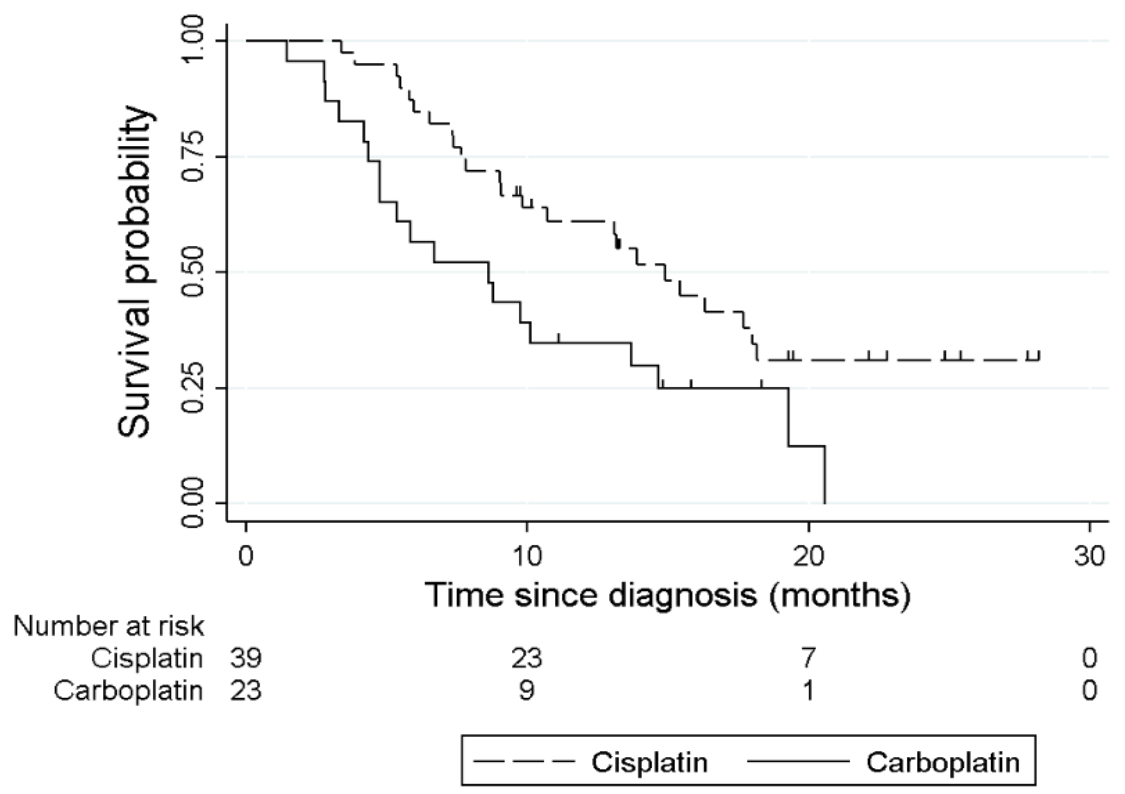

Figure 3. Survival Curve Comparing Cisplatin and Carboplatin-Based Chemotherapy in Fit Patients 
for frailty syndrome, vice versa; frailty is the risk for postoperative morbidity and worse outcome (Wagner et al., 2016; Gani et al., 2017). Therefore, there should be screening for frailty as a preoperative risk assessment.

Obesity is the main risk factor for frailty. For each $1 \mathrm{mg} / \mathrm{m}^{2} \mathrm{BMI}$ increase, risk factors for frailty increased by 1.3 times. This result is supported by many published data on community-dwelling adults (Sheehan et al., 2013). Even though high-fat mass is another factor indicating frailty, it is not an independent factor after adjustments are made for other factors. These data suggest that obesity is important in frailty but not only just high-fat mass. There are other factors or consequences of obesity leading to the inability to adjust to stressors such as nutritional status, physical activity, inflammation that were not measured in this study.

Frailty is associated with a worse survival outcome in patients with various malignancies (Handforth et al., 2015). However, it is not a risk factor for survival in biliary tract cancer with good ECOG performance score. The aggressiveness of the tumor and poor response to chemotherapy and low prevalence of frailty might be the potential causes.

From the pooled analysis, platinum-based chemotherapy with gemcitabine resulted in the highest response rate (Glimelius et al., 1996). Cisplatin and gemcitabine chemotherapy is currently the standard regimen for improved survival compared to gemcitabine alone for advanced biliary tract cancer (Valle et al., 2010; Valle et al., 2016). However, there is no headto-head comparison between cisplatin-based and carboplatin-based regimen. In this study, we found that in fit patients, those who received cisplatin-based chemotherapy resulted in better survival outcome compared to the carboplatin-based regimen. On the contrary, the difference in mortality between the two regimens was not observed among frail patients. Given that cisplatin has more side effects; we would suggest cisplatin for only "fit" patients.

The strength of this study was that all of the patients were biliary tract cancer and ECOG performance status of $0-1$, reducing confounding from primary tumor and performance status which are important risk factors for frailty. The results of this study, however, would be more notable if the nutritional status and frailty assessment before liver surgery were measured.

In conclusion, frailty is prevalent among biliary tract cancer patients receiving chemotherapy. Old age, high BMI, and history of liver surgery were associated with frailty syndrome. Elderly patients should be carefully evaluated for frailty syndrome in addition to performance status.

\section{Disclosure}

The author reports no conflicts of interest in this work.

\section{Acknowledgments}

This project was supported by the Research Grant for New Scholar co-funded by the Office of the Higher Education Commission, TRF and Khon Kaen University
(KKU) (MRG-5980172). We would like to thank Sleep Apnea Research Group, Research Center in Back, Neck and Other Joint Pain and Human Performance, Research and Training Center for Enhancing Quality of Life of Working Age People, and Research and Diagnostic Center for Emerging Infectious Diseases (RCEID), Khon Kaen University, Khon Kaen, Thailand.

\section{References}

Abellan van Kan G, Rolland Y, Bergman H, et al (2008). The I.A.N.A Task Force on frailty assessment of older people in clinical practice. J Nutr Health Aging, 12, 29-37.

Angulo J, El Assar M, Rodriguez-Manas L (2016). Frailty and sarcopenia as the basis for the phenotypic manifestation of chronic diseases in older adults. Mol Aspects Med, 50, 1-32.

Collard RM, Boter H, Schoevers RA, et al (2012). Prevalence of frailty in community-dwelling older persons: a systematic review. J Am Geriatr Soc, 60, 1487-92.

Courtney-Brooks M, Tellawi AR, Scalici J, et al (2012). Frailty: an outcome predictor for elderly gynecologic oncology patients. Gynecol Oncol, 126, 20-4.

Ethun CG, Bilen MA, Jani AB, et al (2017). Frailty and cancer: Implications for oncology surgery, medical oncology, and radiation oncology. CA Cancer J Clin, 67, 362-77.

Fried LP, Tangen CM, Walston J, et al (2001). Frailty in older adults: evidence for a phenotype. J Gerontol A Biol Sci Med Sci, 56, M146-56.

Gani F, Cerullo M, Amini N, et al (2017). Frailty as a risk predictor of morbidity and mortality following liver surgery. J Gastrointest Surg, 21, 822-30.

Glimelius B, Hoffman K, Sjoden PO, et al (1996). Chemotherapy improves survival and quality of life in advanced pancreatic and biliary cancer. Ann Oncol, 7, 593-600.

Handforth C, Clegg A, Young C, et al (2015). The prevalence and outcomes of frailty in older cancer patients: a systematic review. Ann Oncol, 26, 1091-101.

Kojima G, Iliffe S, Taniguchi Y, et al (2017). Prevalence of frailty in Japan: A systematic review and metaanalysis. J Epidemiol, 27, 347-53.

Kristjansson SR, Nesbakken A, Jordhoy MS, et al (2010). Comprehensive geriatric assessment can predict complications in elderly patients after elective surgery for colorectal cancer: a prospective observational cohort study. Crit Rev Oncol Hematol, 76, 208-17.

Kristjansson SR, Rønning B, Hurria A, et al (2012). A comparison of two pre-operative frailty measures in older surgical cancer patients. J Geriatr Oncol, 3, 1-7.

Lee DR, Kawas CH, Gibbs L, et al (2016). Prevalence of frailty and factors associated with frailty in individuals aged 90 and older: The 90+ study. J Am Geriatr Soc, 64, 2257-62.

Sheehan KJ, O'Connell MD, Cunningham C, et al (2013). The relationship between increased body mass index and frailty on falls in community dwelling older adults. $B M C$ Geriatr, 13, 132.

Sookprasert A, Chindaprasert J, Wirasorn K (2012). Systemic therapy for locally advanced and metastatic cholangiocarcinoma. Asian Pac J Cancer Prev, 13, 3-6.

Valle J, Wasan H, Palmer DH, et al (2010). Cisplatin plus gemcitabine versus gemcitabine for biliary tract cancer. N Engl J Med, 362, 1273-81.

Valle JW, Borbath I, Khan SA, et al (2016). Biliary cancer: ESMO Clinical Practice Guidelines for diagnosis, treatment and follow-up. Ann Oncol, 27, 28-37.

Wagner D, DeMarco MM, Amini N, et al (2016). Role of frailty and sarcopenia in predicting outcomes among patients 
DOI:10.31557/APJCP.2019.20.5.1497

undergoing gastrointestinal surgery. World J Gastrointest Surg, 8, 27-40.

\section{(c) (i) (8)}

This work is licensed under a Creative Commons AttributionNon Commercial 4.0 International License. 